\title{
The Standardization of the Translation of "Action and Indications" in the Traditional Chinese Patent Medicine Instruction
}

\author{
Baoxia Zuo \\ Tianjin Tianshi College, Tianjin, 301700, China
}

\begin{abstract}
Keywords: Traditional Chinese Medicine; Action and indications; Terminology; Translation; Standard; Strategy
\end{abstract}

\begin{abstract}
The translation of TCM terms is associated with the pharmaceutical industry's future development. And it is associated with the occupation of the overseas markets, and it is associated with whether it can attract foreign customers to pay attention to traditional Chinese Patent medicine. Therefore, the English translation of the "Action and Indications" of the traditional Chinese Patent medicine is particularly important and essential. And it is also a new research field and direction. In this paper, we explore the English translation of the "Action and Indications" of the traditional Chinese Patent medicine. And we summarize language style characteristics of the "Action and Indications" of the traditional Chinese Patent medicine. And we analyze the current principles of the translation of TCM terms. Furthermore, we list the existing problems of the English translation of the "Action and Indications" of the traditional Chinese Patent medicine. In main purpose of this paper is to point out some effective translation methods and strategies for the English translation of the "Action and Indications" of the traditional Chinese Patent medicine.
\end{abstract}

\section{Introduction}

For the functions and indications in the Chinese medicine instructions (CMI), it is based on the theory of Chinese medicine syndrome differentiation to guide the general description of clinical medicine, reflecting the Chinese medicine syndrome differentiation, the different scientific systems of Chinese medicine and modern medicine, and the level of modernization of Chinese medicine discipline itself. Its logicality and preciseness on the form of word expression reflects the scientificity of the traditional Chinese pharmacology itself. Currently, the word expression and English translation of the functions and indications in the specifications of Chinese patent medicine is not uniform; therefore, it is of great significance for the standardization of word expression and unification of the English translation for the functions and indications in the specifications of Chinese patent medicine. TCM terms integrate the Chinese Taoism and the theories in the "Book of Changes", emphasizing the mutual balance, interdependence, mutual restraint and mutual transformation of everything in the world. These theories themselves are mostly derived from the inference and imagination of ancient people on the natural and human physiological phenomenon, reflecting in the expression of traditional Chinese medicine is relatively subjective, and lack of the support and objective scientific basis and test data. The specifications of Chinese patent medicine still use a lot of Chinese medicine terms; therefore, the translation quality of these terms will directly affect the core information transmitted from the indications and functions of drugs. Therefore, it has a very important practical significance for the translation methods and strategic research in studying the functions and indications in the specifications of Chinese patent medicine.

\section{Wording Characteristics of Functions and Indications Vocabulary in the Specifications of Chinese Patent Medicine}

\section{Frequent use of four-character structure}

In the specifications of Chinese patent medicine, the functions and indications terms of Chinese medicine concerning the descriptions of etiology mechanism, treatment principles and indications diseases have followed the language of the ancient Chinese medicine, which all use four-character 
structures. Four-character structure is readable, and is suitable for the simple features of specifications. Four-character structure is concise and comprehensive, which is able to use extremely refined language to express rich meanings. If it translates according to the literal meaning, each four-character word needs to translate into one or two English sentences, easily leading to lengthy translation.

\section{Vague wording and lack of professionalism}

Our traditional medicine is based on the Chinese philosophy and Taoism, thereby forming the viscera doctrine, yin and yang theory, blood theory and Chinese Herbs doctrine with Chinese characteristics. However, the concept and health care system of Chinese medicine is lack of strong support for scientific experiments, mainly diagnosing through the subjective observation, reasoning and experience of medical practitioners. Therefore, the language of Chinese medicine is abstract and vague, leading to many TCM terms are lack of corresponding English vocabulary. Jiaxu Chen said, "TCM term itself is not standardized, polysemy, with unclear connotation and uncertain extension.”

\section{Polysemy and generalized language}

In TCM terms, there are many wordings have more than one means of interpretation, namely many terms are expressed the state of polysemy. For example, for the term "fever", it has "strong fever", "low fever", "hectic fever" and "hiding fever", but it is more difficult to distinguish, for there is no specific standard of temperature, so it is very different to unify the translation of TCM terms.

\section{Frequent use of parallelism antithesis sentence}

The parataxis characteristic of Chinese determines that the writing style of the specifications of Chinese patent medicine will omit some function words such as conjunctions, prepositions; in addition, in the specification of Chinese patent medicine, it will largely use four-character words, which has neat and orderly antithesis, giving a coherent parallelism momentum. Meanwhile, English emphasizes on the form, and how to translate the original text under the premise of simplicity and accuracy without violating the rules of English grammar is very difficult.

\section{The Principles of English Translation for the Functions and Indications in the Specification of Chinese Patent Medicine}

\section{Principle of naturalness}

The translated terms should be the natural equivalent expression of the target language; precisely speaking, the concepts in the TCM terms closed to Western medicine can use the corresponding Western terminologies to translate.

\section{Principle of conciseness}

Translation needs to be concise and to the point, and the most important feature of TCM term is concise and comprehensive. Professor Li Guozhao once has proposed the information density principle to test the conciseness of translation, and put forward the reference formula of information density:

Information density = Number of meaningful units (notional words) in the original text/Number of meaningful units (notional words) in the translation

This information density is divided into three grades: Grade A is 0.5 , Grade B is 0.25 , and Grade C is 0.1 . The best information density is not lower than Grade A, the words lower than Grade B needs repeated deliberation, and the words lower than Grade B should not be adopted.

\section{Principle of nationality}

The unique medicine system of TCM has Chinese characteristics, and these characteristics in the terms of Western medicine can not find equivalent words; therefore, the English translation of these terms must comply with the principle of nationality.

\section{Principle of back-translation}

The translated Chinese medicine terms should structurally similar to the Chinese form, and if does it like this, the Chinese medicine in the international exchange can better achieve the mutual transmission of information. 


\section{Principle of prescription}

For the translation of TCM terms, it should be regulated in the connotation, so that it can not have other explanations, and the purpose is to eliminate the potential understandings.

\section{Existing Problems of English Translation for the Functions and Indications in the Specifications of Chinese Patent Medicine}

At present, one of the main factors affecting the overseas sales of Chinese patent medicine is that the English translation of functions and instructions in the specifications of Chinese patent medicine appears a series of problems, and the followings are some of the problems appeared in the functional words translation:

\section{Lengthy and miscellaneous translation}

For this practical style of specification, the first thing is to be clear and concise, and to use the most popular language to tell readers the largest information. While in the words of functions and indications in the specification of Chinese patent medicine, there are full of TCM cultural information, and when translating, many translators only known about English without Chinese medicine basis can only resort to TCM English dictionary. Many translations of TCM terms emphasize on explanatory, and the lengthy expressions do not comply with the language characteristics of the specification.

\section{Under-translation and difficult understanding}

Another extreme in the translation of functional words in Chinese patent medicine is under-translation. In terms of higher degree culture loading, under-translation means that on the processing of much cultural information, it does not completely understand its connotation, and the translation does not meet certain communication effect. And after reading the translation, the readers are still difficult to understand, causing the meaningless translation. For the specification of Chinese patent medicine, under-translation is commonly existed. Therefore, in order to keep the conciseness of translations, the translators would most likely choose not to dispose or simply dispose, which do not fully consider the level of Chinese medicine for Western common readers, resulting in insufficient translation of target language.

\section{English Translation Methods and Strategies for the Functions and Indications in the Specifications of Chinese Patent Medicine}

\section{Equivalence in translation}

Due to there is a relatively big difference between the terms of traditional Chinese medicine and Western medicine, so the terms of functions and indications in the specifications of Chinese patent medicine can not find all the equivalent expressions in the terms of Western medicine. Therefore, it can adopt the way of "Equivalence in translation", that is, for the terms with strict equivalences and exactly the same connotations and denotations of Western medical concepts, they can refer directly to the corresponding Western terms. For example, the functions and indications of the Chinese patent medicine "Hou Ji Ling": clearing away heat and toxic materials, and eliminating turgescence and pains, using for parotitis, amygdalitis, and acute pharyngitis, etc. Using the equivalent translation method, it can be translated into: Actions and indication: antipyretic and antidote, detumescence and aces dyne. It is indicated for parotitis, tonsillitis, acute pharyngitis... Due to the difference in Eastern and Western cultures, only a small part of the terms of Chinese medicine in the "indications" of Chinese patent medicine can adopt “Equivalence in translation”, for example: 心烦意乱: restless; 失 眠多梦: insomnia and dreaminess; 不思饮食:losing appetite.

\section{Equivalence value in translation}

For the four-character Chinese medicine terms, if when using the literal translation can not accurately express the whole meaning of the original terms, it can adopt the interpretive translation method, that is Equivalence value in translation. The terms of functions and indications for the 
specifications of Chinese patent medicine translate into "noun phrase", "verb phrase" and "gerund phrase”.

(1) Translate into noun phrase

For example:

气郁不舒: uncomfortable feeling due to the stasis of vital energy

头晕自汗: dizziness and night sweat

气血两虚: deficiency of vital energy and blood

(2) Translate into verb phrase

For example:

消积化滞: promote digestion to relieve dyspepsia

补气养血: invigorate the vital energy to nourish the blood

行气止痛: regulate vital energy circulation to relieve pain

(3) Translate into gerund phrase

For example:

“蜜炼川贝枇杷膏”的功能与主治：清热润肺，止咳平喘，理气化痰”

Translate into: Actions and indication: clearing away heat and moisturizing lungs, stopping cough and asthma, regulating $q i$ and removing sputum.

\section{Transliteration}

With the exchanges and interactions between Eastern and Western cultures, some new English words of medicine have gradually become the widely known vocabulary in people's daily life and work. These new words appeared in English itself without translating into Chinese, so does the terms of Chinese medicine. For example, the "qi" in Chinese medicine has the functional qi, material qi, inborn qi, acquired qi, primordial qi, pectoral qi, defensive qi, kidney qi, middle qi, etc., which can be directly replaced with pinyin “qi”. And another example: 阴阳: yinyang; 五行: wuxing; 推拿:tuina; 三焦: sanjiao, and so on. This can make the unique concept of Chinese medicine keep its unique connotation in the English translation.

\section{Conclusion}

The translation of functions and indication for the specification of Chinese patent medicine is the key to the entire specification. In order to overcome the communication barriers in the difference between Chinese and Western medical treatment, and in order to enable the Western consumers can quickly, easily and accurately understand the core information transmitted from the functions and indications of Chinese patent medicine, and finally buy the drugs, under the premise of the absence of any Chinese medical culture basis, the translators need to focus on the cultural and communicative level of translation, boldly conduct appropriate deletion, interpretation and revision, so that to achieve the communications and exchanges, and to contribute to the ultimate goal of successful sales of Chinese patent medicine.

\section{Acknowledgments}

This paper is one of the achievements for the Humanities and Social Sciences projects in Tianjin's Colleges and Universities, and the project number is: 20142220.

\section{References}

[1] Cai Suqin. Vocabulary and Syntactic Features of the English Specifications of Drugs and Its Translation. Journal of Ningbo University (Educational Science Edition). 2006 (01)

[2] Fan Ye. Discussion the Translation of Specifications from the Prospective of Skopos Theory. Social Sciences Review (New Theory Edition). 2008 (02) 
[3] He Jian, Chen Jiansheng. Discussion on the Literary Form and Translation from the Prospective of Grammatical Metaphor. Journal of Changsha Railway University (Social Science). 2008 (01)

[4] Li Zhaoguo. Introduction of Traditional Chinese Medicine Translation. Lanzhou: Northwest University Press, 1993.

[5] Li Zhaoguo, Zhu Zhongbao. English Translation Kills of Chinese Traditional Medicine. Beijing: People’s Medical Publishing House, 2001:58-60.

[6] Li Junmei. Lexical Vacancy and Translation of TCM Terms. Chinese Journal of Integrated and Western Medicine, 2007, 27(3): 272-273.

[7] Li Shujie. Three Rules of Teleology and Translation of the Specification of Drugs. Journal of Liaoning Medical University (Social Science Edition). 2008 (04)

[8] Luo Haiyan, Shi Yunzhong. Basic Problem and Strategy of English Translation for the Specification of Chinese Medicine. Journal of Traditional Chinese Medicine. 2009(01)

[9] Xiao Qiong. Translation of the Specifications of Chinese Patent Medicine - Communicative Translation Method [D]. Master's Thesis of Guangdong University of Foreign Studies, 2008.

[10]Xiao Yanjiao, Ye Jing. Translation of English Specification of Drugs. Scientific and Technological Information. 2009 (05)

[11]11. Zhang Jing. Discussion on the Translation Criteria from the Specification of Drugs and Cosmetic. Journal of Henan Business College. 2005 (02) 